\title{
DSM-IV Axis II personality disorders and suicide and attempted suicide in China
}

Yongsheng Tong, Michael R. Phillips and Kenneth R. Conner

\section{Background}

There are meagre data on Axis II personality disorders and suicidal behaviour in China.

\begin{abstract}
Aims
To describe the prevalence of Axis II personality disorders in suicides and suicide attempts in China and to estimate risk for these outcomes associated with personality disorders.

\section{Method}

People who died by suicide $(n=151)$, people who attempted suicide $(n=118)$ and living community controls $(n=140)$ were randomly sampled from four chinese counties and studied using the Structured Clinical Interviews for DSM-IV-TR Axis I Disorders (SCID-I) and Axis II Personality Disorders (SCID-II). We also determined the prevalence of subthreshold versions of ten DSM-IV personality disorders.
\end{abstract}

\section{Results}

Axis II personality disorders were present in $7 \%$ of the suicide group, $6 \%$ of the suicide attempt group and $1 \%$ of the control group. Threshold and subthreshold personality disorders had adjusted odds ratios (point estimates) in the range of 2.7-8.0 for suicide and for suicide attempts.

\section{Conclusions}

Axis II personality disorders may confer increased risk for suicidal behaviour in China, but their low prevalence in the community and among people with suicidal behaviour suggests that other personality constructs such as select dimensional traits may be a more fruitful avenue for understanding and preventing suicide in China.

\section{Declaration of interest}

None.

\section{Copyright and usage}

(c) The Royal college of Psychiatrists 2016.
China had a high suicide rate in the mid-1990s ${ }^{1,2}$ that has decreased substantially over the past two decades, but it remains a major public health problem, accounting for approximately 200000 deaths per year. ${ }^{2}$ There are no nationally representative data available on suicide attempt rates, but one previous report estimated an attempt rate in rural parts of the country equal to sixfold the suicide rate. ${ }^{3}$ Epidemiological studies report that $48-63 \%$ of people who died by suicide $e^{4,5}$ and $39 \%$ of people who attempt suicide in China have one or more mental disorders, ${ }^{6}$ much lower prevalence figures than the $90 \%$ and $80 \%$, respectively, reported in high-income countries. ${ }^{7-10}$ Nonetheless, controlled studies in China have shown that mental disorders are potent risk factors for both suicide attempt ${ }^{6}$ and suicide, ${ }^{4,5}$ and some data suggest that mental disorders are the most important risk factor for suicidal behaviour in China. ${ }^{11}$ In most countries and regions where research on personality disorders and suicidal behaviour have been conducted, personality disorders are relatively common among people with suicidal behaviour. ${ }^{8,9}$ In Europe, estimates are that $46-74 \%$ of people who attempt suicide ${ }^{12-14}$ and $32-71 \%$ of people who die by suicide meet diagnostic criteria for one or more personality disorders. ${ }^{14-19}$ In North America, one study found that $65 \%$ of people who attempted suicide ${ }^{20}$ had personality disorder(s); and another study reported that $57 \%$ of young suicides $^{21}$ had Axis II personality disorders. ${ }^{22}$ Despite their relatively high prevalence in people who die by suicide (and attempt suicide), the magnitude of risk for suicidal behaviour associated with personality disorder may be lower than that associated with Axis I mental disorders. ${ }^{15-17,19}$ Nonetheless, available data support the idea that personality disorders confer risk beyond that attributed to Axis I disorders. ${ }^{21}$ Moreover, a study of attempted suicide estimated that personality disorders account for $30 \%$ of the population attributable risk for attempted suicide, highlighting the importance of targeting personality disorders in prevention efforts. ${ }^{20}$
Available data show that the combination of Axis I mental disorders and Axis II disorders are common among those who die by suicide $16,17,19,23,24$ and among those who attempt suicide. $^{7,25}$ The presence of comorbid Axis I mental disorders and Axis II personality disorders may be associated with a particularly high risk for attempted suicide ${ }^{12}$ and suicide. ${ }^{16,17,19,24}$ Previous studies reported that personality disorders associated with affective lability and impulsivity (included in cluster B personality disorders in the DSM classification system ${ }^{22}$ and under the 'emotionally unstable personality disorder' in the ICD system $^{26}$ ) had a higher prevalence among those who died by suicide $^{19,21,24}$ and those who attempted suicide ${ }^{7,20,25}$ than other types of personality disorders. Studies using the DSM system suggest that individuals with borderline personality disorder are also at a particularly high risk for attempted suicide ${ }^{27-29}$ or suicide $^{30}$ Although those who die by suicide and those who attempt suicide often differ demographically, particularly in Western studies, there is substantial overlap in the pattern of their reported personality disorders. ${ }^{31}$ Most studies on Axis II personality disorders and suicide or suicide attempt have been conducted in Europe, North America or in Asia Pacific regions other than mainland China. There is only one study that has focused on personality disorders and suicide conducted in Taiwan, ${ }^{24}$ but the relevance of these results to mainland China is unclear. The purpose of the current study is to address the gaps in data on personality disorders and suicidal behaviour in China. More specifically, we compared the prevalence of Axis I and Axis II mental disorders in individuals who died by suicide, attempted suicide and non-suicidal community controls in China, and assessed the potential role of personality disorders as independent risk factors for suicide and attempted suicide. In a broader context, the study also aimed to clarify the targets for prevention of suicidal behaviour in China, with a focus on whether or not Axis II personality disorders should be prioritised. 


\section{Method}

\section{Sampling}

The enrolment process for the study is shown in Fig. 1. One rural county from each of four provinces in China (Shaanxi, Sichuan, Hebei and Inner Mongolia) was selected for the study. The death certificate reporting systems of the four counties (managed by the local offices of the national centres for disease control) were used to identify consecutive decedents 18 years of age and older with the cause of death listed as suicide (ICD-10 codes X60-X84), other injury (ICD-10 codes V01-X59) or mental illness (ICD-10 codes F00-F98) that occurred from January 2008 to March 2011 in Inner Mongolia, from January 2008 to June 2009 in Hebei, from June 2008 to December 2010 in Shaanxi and from June 2008 to March 2011 in Sichuan (the suicide group). ${ }^{26}$ We included those for whom the death certificate identified 'mental illness' as the cause of death because our previous studies found that many of these individuals had died by suicide. Injury deaths were assessed as deceased controls (not reported in this paper), but if detailed investigation found that the injury death should be reclassified as a suicide, then it was included in the suicide group sample reported in this paper.

We established a suicidal behaviour register system in the emergency departments of general hospitals in the four target counties. All patients aged 18 and older who were treated for suicidal behaviour in these hospitals during the study period were potential participants in the study (the suicide attempt group). Those who died in the hospital - some of whom did not get reported as suicide on the death certificate - were categorised as suicide decedents.

We used 2008 census data for three of the four counties (the county in Hebei Province stopped participating in the study in 2009), to select a multistage stratified random sample of adult (i.e. $\geqslant 18$ years of age) community residents as the living controls (the control group).

For each identified individual in the suicide, suicide attempt and control groups, one family member proxy respondent and one other proxy respondent (friend, neighbour) were interviewed separately by trained interviewers. Participants in the suicide attempt and control groups were also interviewed themselves by a third trained interviewer. The interviews lasted $2-3 \mathrm{~h}$. Proxy respondents of those in the suicide group were interviewed within 12 months of death and participants in the suicide attempt group and their proxy respondents were interviewed within 6 months of the index attempt. The study was approved by the institutional review board of the Beijing Hui Long Guan Hospital and written informed consent was obtained from all living participants.

\section{Measures}

The Chinese version of the Structured Clinical Interview for DSM-IV (SCID-I/P) ${ }^{32}$ (adapted for use with proxy informants) was administered by a psychiatrist to determine the presence or absence of current Axis I mental disorders. Axis II personality disorders were based on the Chinese version of the Structured Clinical Interview for DSM-IV Axis II Personality Disorders (SCID-II), ${ }^{33}$ a structured questionnaire validated in China ${ }^{34}$ that generates diagnoses for ten specific DSM-IV personality disorders. Multiple comorbid Axis I and II disorder diagnoses were made if appropriate. We used a 'rate-up' strategy; that is, if an Axis I or Axis II disorder was identified in either proxy interview, it was coded as present.

To improve the sensitivity of the study to detect personality disorder psychopathology, we also applied subthreshold criteria for the ten specific DSM-IV personality disorders. ${ }^{13,35}$ The subthreshold criteria used in the present study were as follows: three symptoms for schizotypal, borderline, histrionic, narcissistic or dependent personality disorder (five required for threshold); two symptoms for paranoid, schizoid, avoidant or obsessivecompulsive personality disorder (four required for threshold); or two section A symptoms for antisocial personality disorder (three required for threshold).

\section{Statistical analysis}

Analyses are based on multivariate logistic regression for comparisons between the suicide attempt and control groups (based on self-report data) and on multinomial regression for three-way comparisons among those in the suicide, suicide attempt and control groups (based on proxy data). For all comparisons, we ran two sets of models: the first model adjusted for age, gender, marital status (currently married $v$. not currently married), years of formal education and employment status (agricultural labourer, non-agricultural labourer and unemployed or retired); the second model adjusted additionally for Axis I mental disorders. Different sets of models assessed the risk of suicide and suicide attempt in individuals with (a) any personality disorder, (b) any subthreshold personality disorder, (c) subthreshold cluster A (i.e. paranoid, schizoid and schizotypal) personality disorders, (d) subthreshold cluster B (antisocial, borderline, histrionic and narcissistic) personality disorders and (e) subthreshold cluster C (avoidant, dependent and obsessivecompulsive) personality disorders. As a result of the low prevalence of the personality disorders that met full diagnostic criteria, there was insufficient data to analyse risk for suicidal behaviour associated with cluster A, B or C personality disorders or any specific personality disorders.

\section{Results}

Data from 151 suicides, 118 suicide attempts and 140 controls were analysed. Co-resident family members completed interviews for 145 (96\%) individuals in the suicide group, 113 (96\%) in the suicide attempt group and 139 (99\%) in the control group; other proxy informants (friends or acquaintances) completed interviews for $151(100 \%)$ in the suicide group, $114(97 \%)$ in the suicide attempt group and 139 (99\%) in the control group. The participants themselves completed interviews, 107 (91\%) in the suicide attempt group and 128 (91\%) in the control group.

Table 1 shows the demographic characteristics of the three groups. The suicide attempt group were younger and more likely to be female than the other groups. The suicide group had less education and were more likely to be unmarried than the other groups. There were no statistically significant differences in demographic characteristics between the 107 participants in the suicide attempt group who personally completed the interview and the 11 who did not complete it. However, the 12 participants in the control group who did not complete the interview were less educated, older and more likely to be unmarried than the 128 who completed the interview.

Table 2 shows the prevalence of any Axis I mental disorder and of the full-criteria and subthreshold-criteria Axis II personality disorders as reported by proxy informants (whole sample) and as reported by the participant directly (suicide attempt and control groups). Based on proxy reports, the prevalence of any personality disorder was much lower than the prevalence of any Axis I mental disorder in the suicide group (6.6\% v. 68.2\%), suicide attempt group $(5.9 \%$ v. $32.2 \%)$ and the control group $(0.7 \%$ v. $3.6 \%)$. In total, $80 \%$ of the suicide group with any Axis 


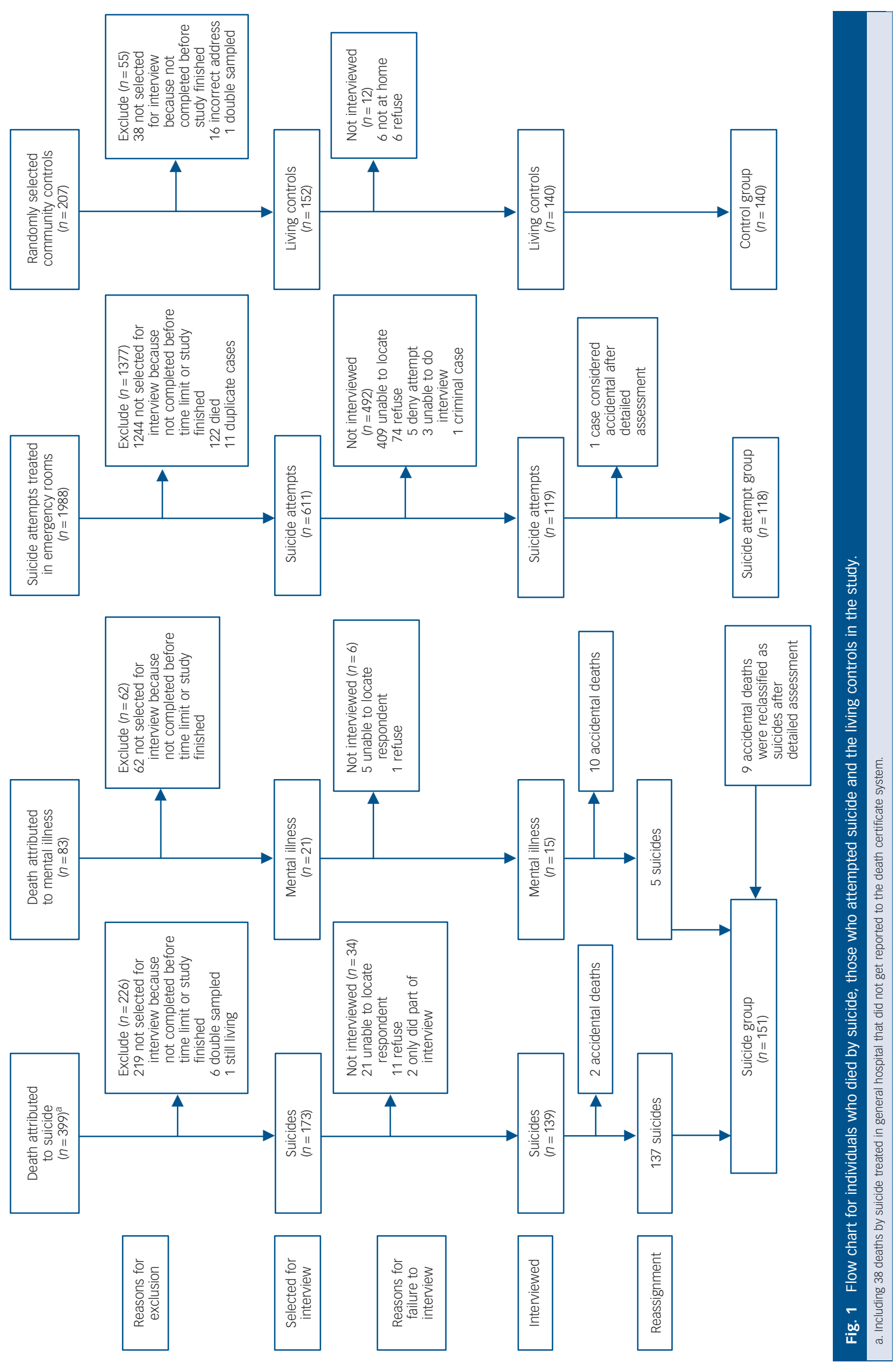




\begin{tabular}{|c|c|c|c|c|c|c|c|}
\hline & $\begin{array}{l}\text { Suicide group } \\
\qquad(n=151)\end{array}$ & $\begin{array}{l}\text { Suicide attempt } \\
\text { group }(n=118)\end{array}$ & $\begin{array}{l}\text { Control group } \\
\quad(n=140)\end{array}$ & $F$ & $\chi^{2}$ & $P$ & Multiple comparisons \\
\hline Age, years: mean (s.d.) & $51.1(18.0)$ & $40.3(13.0)$ & 49.7 (13.9) & 16.64 & & $<0.001$ & Suicide attempt group < suicide group, control group \\
\hline $\begin{array}{l}\text { Years of formal education, } \\
\text { mean (s.d.) }\end{array}$ & $4.8(3.6)$ & $6.3(3.1)$ & $6.2(3.4)$ & 9.32 & & $<0.001$ & Suicide group < suicide attempt group, control group \\
\hline Female, $n(\%)$ & $68(45.0)$ & $78(66.1)$ & $65(46.4)$ & & 14.04 & 0.001 & Suicide attempt group > suicide group, control group \\
\hline Currently unmarried, $n(\%)$ & $52(34.4)$ & $13(11.0)$ & $16(11.4)$ & & 32.28 & $<0.001$ & Suicide group > suicide attempt group, control group \\
\hline Employment status, $n$ (\%) & & & & & 8.54 & 0.074 & \\
\hline Agricultural labourers & $85(56.3)$ & $85(72.0)$ & $92(65.7)$ & & & & \\
\hline Non-agricultural labourers ${ }^{a}$ & $27(17.9)$ & $16(13.6)$ & $24(17.1)$ & & & & \\
\hline Unemployed or retired & $39(25.8)$ & $17(14.4)$ & $24(17.1)$ & & & & \\
\hline
\end{tabular}

II personality disorder had comorbid Axis I mental disorder(s) and $71.4 \%$ of the suicide attempt group with any personality disorder had comorbid Axis I disorder(s) (data not shown).

Table 3 shows the adjusted odds ratios (AORs) for the suicide and suicide attempt groups (compared with the control group and with each other) associated with Axis I disorders, any threshold or subthreshold personality disorder, and subthreshold cluster A, B and $\mathrm{C}$ personality disorders, after adjustment for demographic variables (Model 1) and after additional adjustment for Axis I mental disorders (Model 2). The Model 1 analyses show that after adjusting for age, gender, years of formal education, marital status and employment status:

(a) any personality disorder, any subthreshold personality disorder, and subthreshold cluster A and $\mathrm{C}$ personality disorders are associated with risk for suicide;

(b) any subthreshold personality disorder and all three subthreshold personality disorder clusters are associated with risk for attempted suicide;

\begin{tabular}{|c|c|c|c|c|c|c|c|c|c|c|}
\hline \multirow[b]{3}{*}{ DSM-IV disorders } & \multicolumn{6}{|c|}{ Proxy-based data } & \multicolumn{4}{|c|}{ Self-report data ${ }^{a}$} \\
\hline & \multicolumn{2}{|c|}{$\begin{array}{l}\text { Suicide group } \\
\qquad(n=151)\end{array}$} & \multicolumn{2}{|c|}{$\begin{array}{l}\text { Suicide attempt group } \\
\qquad(n=118)\end{array}$} & \multicolumn{2}{|c|}{$\begin{array}{l}\text { Control group } \\
\quad(n=140)\end{array}$} & \multicolumn{2}{|c|}{$\begin{array}{l}\text { Suicide attempt group } \\
\qquad(n=107)\end{array}$} & \multicolumn{2}{|c|}{$\begin{array}{l}\text { Control group } \\
\qquad(n=128)\end{array}$} \\
\hline & $n$ & $\%$ & $n$ & $\%$ & $n$ & $\%$ & $n$ & $\%$ & $n$ & $\%$ \\
\hline Any Axis I mental disorders & 103 & 68.2 & 38 & 32.2 & 5 & 3.6 & 47 & 43.9 & 10 & 7.8 \\
\hline Any personality disorders & 10 & 6.6 & 7 & 5.9 & 1 & 0.7 & 7 & 6.5 & 1 & 0.8 \\
\hline Cluster A & 3 & 2.0 & 2 & 1.7 & 1 & 0.7 & 2 & 1.9 & 0 & 0.0 \\
\hline Paranoid & 1 & 0.7 & 0 & 0.0 & 0 & 0.0 & 0 & 0.0 & 0 & 0.0 \\
\hline Schizoid & 2 & 1.3 & 2 & 1.7 & 1 & 0.7 & 2 & 1.9 & 0 & 0.0 \\
\hline Schizotypal & 0 & 0.0 & 0 & 0.0 & 0 & 0.0 & 0 & 0.0 & 0 & 0.0 \\
\hline Cluster B & 1 & 0.7 & 1 & 0.8 & 0 & 0.0 & 1 & 0.9 & 0 & 0.0 \\
\hline Antisocial & 0 & 0.0 & 0 & 0.0 & 0 & 0.0 & 0 & 0.0 & 0 & 0.0 \\
\hline Borderline & 1 & 0.7 & 1 & 0.8 & 0 & 0.0 & 1 & 0.9 & 0 & 0.0 \\
\hline Histrionic & 0 & 0.0 & 0 & 0.0 & 0 & 0.0 & 0 & 0.0 & 0 & 0.0 \\
\hline Narcissistic & 0 & 0.0 & 0 & 0.0 & 0 & 0.0 & 0 & 0.0 & 0 & 0.0 \\
\hline Cluster C & 6 & 4.0 & 4 & 3.4 & 0 & 0.0 & 5 & 4.7 & 1 & 0.8 \\
\hline Avoidant & 3 & 2.0 & 4 & 3.4 & 0 & 0.0 & 3 & 2.8 & 1 & 0.8 \\
\hline Dependent & 0 & 0.0 & 0 & 0.0 & 0 & 0.0 & 0 & 0.0 & 0 & 0.0 \\
\hline Obsessive-compulsive & 3 & 2.0 & 0 & 0.0 & 0 & 0.0 & 2 & 1.9 & 0 & 0.0 \\
\hline \multicolumn{11}{|l|}{$\begin{array}{l}\text { Combining threshold and } \\
\text { subthreshold diagnoses }\end{array}$} \\
\hline Any personality disorders & 41 & 27.2 & 27 & 22.9 & 8 & 5.7 & 19 & 17.8 & 11 & 8.6 \\
\hline Cluster A & 17 & 11.3 & 17 & 14.4 & 3 & 2.1 & 8 & 7.5 & 2 & 1.6 \\
\hline Paranoid & 6 & 4.0 & 8 & 6.8 & 0 & 0.0 & 5 & 4.7 & 1 & 0.8 \\
\hline Schizoid & 13 & 8.6 & 13 & 11.0 & 3 & 2.1 & 6 & 5.7 & 1 & 0.8 \\
\hline Schizotypal & 5 & 3.3 & 1 & 0.8 & 0 & 0.0 & 0 & 0.0 & 1 & 0.8 \\
\hline Cluster B & 8 & 5.3 & 9 & 7.6 & 1 & 0.7 & 3 & 2.8 & 0 & 0.0 \\
\hline Antisocial & 4 & 2.6 & 4 & 3.4 & 0 & 0.0 & 0 & 0.0 & 0 & 0.0 \\
\hline Borderline & 4 & 2.6 & 4 & 3.4 & 0 & 0.0 & 2 & 1.9 & 0 & 0.0 \\
\hline Histrionic & 3 & 2.0 & 1 & 0.8 & 0 & 0.0 & 0 & 0.0 & 0 & 0.0 \\
\hline Narcissistic & 1 & 0.7 & 2 & 1.7 & 1 & 0.7 & 1 & 0.9 & 0 & 0.0 \\
\hline Cluster C & 30 & 19.9 & 15 & 12.7 & 6 & 4.3 & 13 & 12.1 & 10 & 7.8 \\
\hline Avoidant & 11 & 7.3 & 8 & 6.8 & 2 & 1.4 & 9 & 8.4 & 4 & 3.1 \\
\hline Dependent & 0 & 0.0 & 1 & 0.8 & 0 & 0.0 & 0 & 0.0 & 0 & 0.0 \\
\hline Obsessive-compulsive & 23 & 15.2 & 10 & 8.5 & 4 & 2.9 & 5 & 4.7 & 6 & 4.7 \\
\hline
\end{tabular}




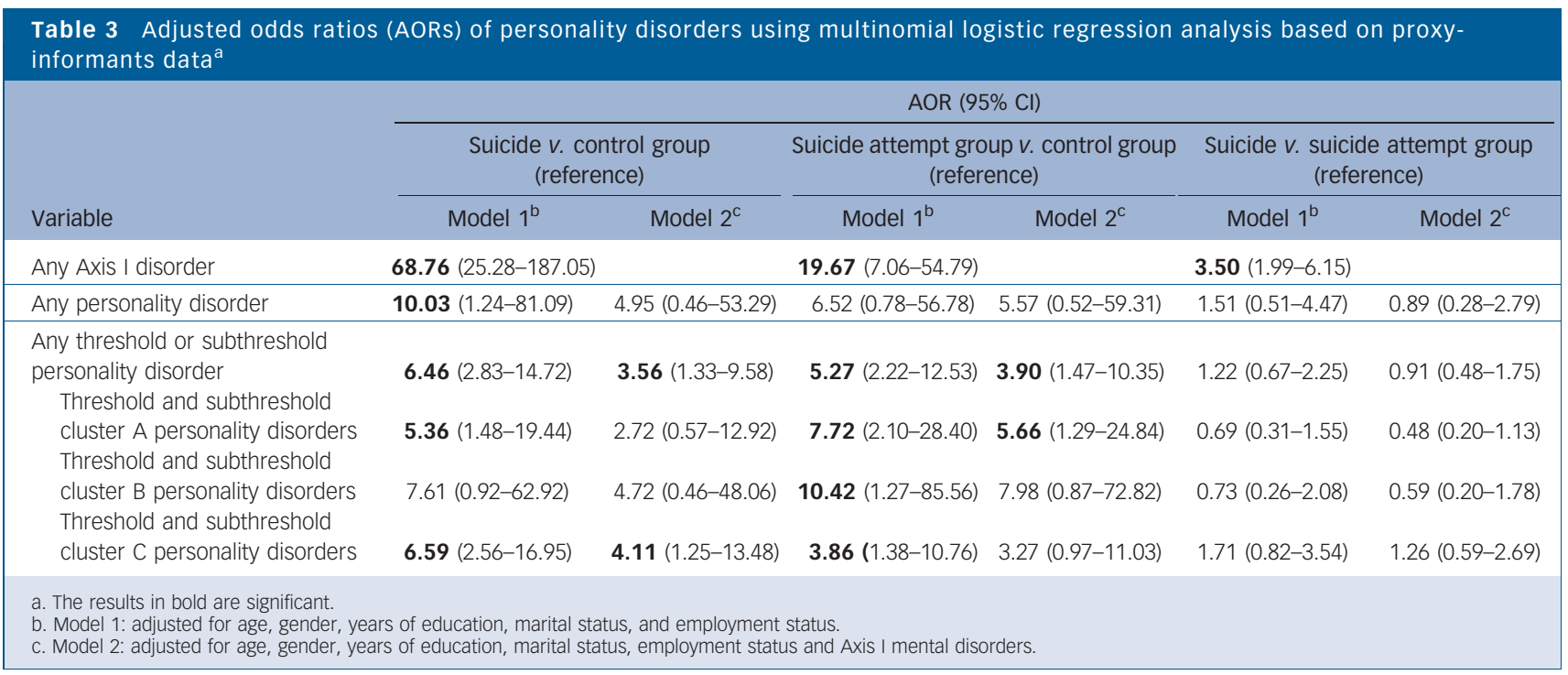

(c) the personality variables that were not associated with suicide (subthreshold cluster B personality disorder) and suicide attempt (any threshold personality disorder) at a statistically significant level had relatively high AORs (point estimates) compared with the control group (7.61 and 6.52 respectively), which suggests that sparse data and corresponding wide confidence intervals contributed to the non-significant results;

(d) the suicide group were more likely to have any Axis I disorder than the suicide attempt group but none of the personality variables differentiated the suicide group from the suicide attempt group; and

(e) in all cases the AOR for any Axis I disorder was much greater than that for any of the personality variables.

In the Model 2 analyses (also shown in Table 3) the AORs are adjusted for both demographic factors and for the presence of any Axis I mental disorder. In all cases, this resulted in a decrease in the magnitude of the AORs compared with Model 1. The AORs for the suicide and suicide attempt groups (compared with the control group) associated with the various personality disorder variables all remained well above 1 (ranging from 2.72 to 7.98 ) but most of them were statistically non-significant. After adjusting for Axis I disorders, only any subthreshold personality disorder and subthreshold cluster $\mathrm{C}$ personality disorder remained statistically significant risk factors for suicide and only any subthreshold personality disorder and subthreshold cluster A personality disorders remained significant risk factors for suicide attempt. Furthermore, the comorbid combination of Axis I disorders and Axis II threshold or subthreshold personality disorders did not confer increased risk for suicide $(\mathrm{AOR}=0.13$, 95\% CI $0.01-1.23$ ) or attempted suicide $(\mathrm{AOR}=0.10,95 \% \mathrm{CI}$ 0.01-1.01), after rigorous adjustment for demographic factors and Axis I and II diagnoses.

Comparison of proxy informant results and self-report results for the suicide attempt and control groups (Table 2) show that the self-reported prevalence of Axis I mental disorders is higher than the prevalence identified through proxy reports, the self-report prevalence of Axis II threshold personality disorders is similar for the two types of informants, and the prevalence of threshold plus subthreshold personality disorders for the suicide attempt group is higher in proxy-based data than in self-report data. However, the prevalence of threshold plus subthreshold personality disorders in the control group is higher in self-report data than in proxy-based data. Based on the self-report results, the Model 1 results show that any personality disorders, any subthreshold personality disorder and subthreshold cluster A personality disorder are associated with increased risk for suicide attempt (Table 4). However, after adjustment for the presence of any Axis I mental disorder in the Model 2 analyses, none of these personality disorder variables remained associated with suicide attempt at a statistically significant level. Similar to proxy-based data, selfreported results did not indicate increased risk for suicide attempt associated with comorbid combinations of Axis I disorders and

Table 4 Adjusted odds ratios (AORs) of personality disorders for the suicide attempt group (compared with the control group) based on self-report data ${ }^{a}$

\begin{tabular}{|c|c|c|}
\hline \multirow[b]{2}{*}{ Variable } & \multicolumn{2}{|c|}{ AOR $(95 \% \mathrm{Cl})$} \\
\hline & Model $1^{\mathrm{b}}$ & Model $2^{c}$ \\
\hline Any Axis I mental disorder & \multicolumn{2}{|l|}{$14.90(6.18-35.91)$} \\
\hline Any personality disorder & $12.57(1.34-118.23)$ & $2.00(0.19-20.50)$ \\
\hline Any threshold or subthreshold personality disorder ${ }^{d}$ & $2.82(1.19-6.71)$ & $0.85(0.28-2.58)$ \\
\hline Threshold and subthreshold cluster A personality disorders & $9.19(1.70-49.62)$ & $3.41(0.43-26.89)$ \\
\hline Threshold and subthreshold cluster $\mathrm{C}$ personality disorders & $1.68(0.66-4.31)$ & $0.47(0.14-1.56)$ \\
\hline \multicolumn{3}{|c|}{$\begin{array}{l}\text { a. The results in bold are significant. } \\
\text { b. Model 1: adjusted for age, gender, years of education, marital status, and employment status. } \\
\text { c. Model 2: adjusted for age, gender, years of education, marital status, employment status, and Axis I mental disorders. } \\
\text { d. AOR of subthreshold cluster B personality disorders was not calculated because, based on the self-report interview, none of the control group had a diagnosis of any cluster B } \\
\text { subthreshold personality disorder. }\end{array}$} \\
\hline
\end{tabular}


Axis II threshold and subthreshold personality disorders ( $\mathrm{AOR}=1.82,95 \%$ CI 0.19-17.57).

\section{Discussion}

\section{Main findings}

In this study, we identified a consecutive series of individuals who died by suicide, a consecutive series of individuals who had attempted suicide and a randomly selected sample of community controls from four localities in China. We independently administered a comprehensive interview - including a structured psychiatric diagnostic examination - to co-resident family informants, to another proxy informant and, for the suicide attempt and control groups, to the individual directly. To ensure that misclassified and missing suicides were included in the sample, we also reclassified deaths recorded as being because of mental illness or accidents as suicides if the detailed $2-3 \mathrm{~h}$ interview indicated that it was a suicide; and we also included 15 deaths from suicide treated in general hospital emergency departments that did not get reported to the local centres for disease control.

We determined that Axis II threshold and subthreshold personality disorders are much less common in those who died by suicide, attempt suicide and in community controls from mainland China than those reported in previous studies from other countries and regions. ${ }^{8-10,12-21,23-25}$ Nevertheless, our results generally suggest that threshold and subthreshold personality disorders confer risk for suicide and suicide attempt. Moreover, adjustment for comorbid Axis I disorders does not eliminate the association between personality disorders and suicidal behaviour, although the association between personality disorders and suicidal behaviour was attenuated, and, in many cases, non-significant.

There were also several negative findings that underline differences in the characteristics of those who engage in suicidal behaviour between China and other countries. Comorbid Axis I and Axis II diagnoses was not associated with a higher risk of suicide or suicide attempt than the corresponding Axis I condition without a comorbid personality disorder. The prevalence of cluster B personality disorders - which includes borderline personality disorder - in the suicide and suicide attempt group was not higher than that of the other personality clusters; in fact, cluster B personality disorders were not significantly associated with attempted suicide. And, despite a higher prevalence of Axis I disorders in the suicide group than in the suicide attempt group, there were no significant differences in the prevalence of any of the threshold and subthreshold personality disorders between these two groups.

Several studies from high-income countries suggest that borderline personality disorder is a prevalent condition among individuals who have suicidal behaviour. ${ }^{19-21}$ However, both the current study and a prior study conducted in mainland China reported a low prevalence of borderline personality disorder among people who died by suicide $(0.7 \%$ in the current study and $1 \%$ in the study by Yang et $\left.a l^{36}\right)$. Even when we expanded the criteria in our study to include individuals with both threshold and subthreshold borderline personality disorder the prevalence remained low, $2.6 \%$ for the suicide group and $3.4 \%$ for the suicide attempt group.

The specific case of borderline personality disorder highlights one of the fundamental questions raised by this study: why is the reported prevalence of DSM-IV personality disorders among community members and among those with suicidal behaviour so low in mainland China compared with reports from other countries? There are several possible explanations: (a) personality disorders as defined by DSM-IV are, in fact, much less prevalent in mainland China; (b) the translated version of the SCID-II instrument is not culturally sensitive enough to identify personality disorders; (c) proxy informants are unaware of or unwilling to report personality abnormalities in their associates; (d) individuals who attempt suicide and controls are unwilling to report personality abnormalities; and (e) clinical interviewers using the SCID-II are not sensitive to nuances in respondents' answers to the structured questionnaire.

The willingness of proxy informants, those who attempted suicide and the controls to report Axis I mental disorders at about the same rate reported in other studies in China suggests that reluctance to report personality characteristics is not the major factor underlying the low reported prevalence. And the SCID-II is a fully structured questionnaire so it is also unlikely that the insensitivity of clinicians who administer the scale resulted in the low reported prevalence. A previous study in a clinical sample of urban residents reported that the Chinese version of the SCID-II had good psychometric properties, ${ }^{34}$ so it is unlikely that poor cultural sensitivity of the instrument resulted in the low rates; but it is possible that its reliability and validity is not as good in community-based samples, particularly those from rural regions (like the current study) so this remains a possible explanation for our findings.

This leaves a final possible explanation: personality disorders as defined by DSM-IV are much less prevalent in mainland China. Personality disorder diagnoses are almost never made in clinical settings in China. It is possible (as suggested by this study) that personality disorders are obscured by Axis I disorders, but individuals with 'free-standing' personality disorders, if they exist in the community, do not commonly seek treatment or get referred for treatment. Community-based studies have identified a variety of 'personality traits' but these almost never get to the level that they significantly impair social or occupational functioning, so they do not meet the stringent DSM-IV entry criteria for a personality disorder. For example, several studies in China have identified both trait and state impulsivity as strong predictors of suicidal behaviour. ${ }^{37,38}$ But individuals with these traits, even when extreme, often start having fluctuating (not chronic) problems in early adulthood (not starting prior to the age of 18) and the problems do not significantly affect their overall social or occupational functioning. Thus, prominent impulsivity is clearly associated with risk for suicidal behaviour but does not fulfil the primary criteria for a personality disorder as defined by DSM-IV.

This brings the discussion around to the ongoing debates about the relative value of using categorical models of personality disorders $v$. using dimensional models of personality traits, ${ }^{13,35,39,40}$ a topic that became a contentious issue during discussions about the role of personality disorders in DSM-5. ${ }^{41}$ Given the results of this study and our clinical experience of working with individuals who are suicidal in China for more than two decades, we are firmly on the side of using dimensional measures of personality traits rather than the dichotomous personality disorder categories of DSM-IV. Adding more culturally sensitive probes may marginally improve the sensitivity of the Chinese version of the SCID-II but, given the basic requirement of significant impairment in social or occupational functioning, it is unlikely that such revisions would substantially increase the reported prevalence of personality disorders in mainland China. Assuming a reliable, valid and feasible method of assessing the severity of personality psychopathology on a limited number of dimensions (traits) can be developed in mainland China (a big assumption), it would be much more useful for evaluating the relationship of personality to suicidal behaviour than the dichotomous DSM-IV personality disorders. 


\section{Strengths and limitations}

This is a relatively large, community-based study that used the same comprehensive structured survey method to obtain information from multiple informants about a wide range of potential risk factors for suicidal behaviour for a sample of people who died by suicide, people who attempted suicide and community controls. This makes it possible to directly compare suicides and attempted suicides, an issue that is particularly important in China where several authors suggest that there is a greater overlap of the two types of suicidal behaviour than is reported in high-income countries. ${ }^{4,6}$ This is, moreover, the first study from mainland China to use a validated structured method to assess the prevalence of personality disorders (and personality traits) among suicide decedents and people who have attempted suicide. However, the study was limited to four rural locations in the country, so it is uncertain whether or not the results would hold true in urban China or in other rural regions of the country.

The study suffers from the methodological problems that are common to all psychological autopsy studies from China. In the absence of previous psychiatric treatment and detailed medical records (very few individuals with mental disorders receive treatment in $\mathrm{China}^{42}$ ) one must depend on the memory and cooperation of proxy informants for information about suicide decedents. Proxy informants may be more likely to accurately identify externalising personality disorders than the individual himself or herself, but they may be less accurate when reporting on internalising personality disorders. More importantly, when using proxy-based information to compare suicide decedents with living controls or to those who have attempted suicide one set of informants has experienced a recent death (in this study about 6 months previously) of the close associate they are discussing, whereas the other set of informants is discussing an individual they have interacted with over the past couple of days. One can try to minimise the effect of these differences by providing extensive training and supervision to interviewers, but it is difficult to completely eliminate potential biases related to differences in informants' experience. One method to reduce these differences is to use accidental death decedents as controls (as was done in the national psychological autopsy study in China conducted from 1996 to $2001^{4}$ ), but this method has the potential disadvantage of obscuring the importance of risk factors that are common to both suicide and accidental deaths. ${ }^{43}$ (The current project, of which this paper is one part, also collected data on an injury death control group, the results of which will subsequently be compared with those of the living control group.)

The study identified a large number of people who died by suicide and people who attempted suicide who were excluded from the study because we did not have the time, funds or personnel to complete the very extensive evaluation of this large number of individuals. There was no systematic bias in the selection of individuals to target for interview, so it is unlikely that this affected the outcome. However, the failure to interview selected individuals, which was a substantial problem in the suicide attempt group, may have biased the sample. Many individuals who get treated for suicide attempts in China provide false names or false addresses in the emergency department (primarily because of fear of stigma) or, if subsequently contacted, deny the suicide attempt or refuse to participate in follow-up evaluations. As a result of these issues we were only able to complete interviews with 119 of the 611 (19\%) selected individuals who had attempted suicide. It is possible that in the suicide attempt group the personality characteristics of those who participated were different from the characteristics of those who we could not interview. On the other hand, the successful interview rate for the stratified random sample of controls was a healthy $92 \%$ (140/152), although the sample only came from three of the four counties included in the study, and non-participants tended to be older, less educated and were less likely to be married. There were no major economic or other differences between the counties, so it is unlikely that failure to select controls from all four sites biased the overall results.

The unexpectedly low prevalence of Axis II personality disorders in the control group (only 1 of the 140 controls meet DSM-IV criteria for any personality disorder) made it difficult to assess the independent effect of specific personality disorders. We tried to minimise this problem by relaxing the rather stringent DSM-IV criteria for personality disorders; but even after using our revised criteria only 8 of the 140 controls $(5.7 \%)$ met criteria for a subthreshold personality disorder. The complete absence of any threshold or subthreshold borderline personality disorder in the control group - the personality disorder that is most closely related to suicide in studies from high-income countries prevented us from assessing its relevance to suicide and suicide attempt in this study. Even when collapsing personality disorders into the three main clusters of disorders, the low prevalence in the control group resulted in very wide confidence limits for the odds ratios, which meant that fairly large AORs (based on point estimates) remained non-significant. Nevertheless, the prevalence of the personality disorders was substantially higher in the suicide and suicide attempt groups than in the control group and the AORs remained high (although diminished) after adjustment for Axis I disorders. And, despite the lower prevalence, these AORs were similar to those reported for personality disorders in studies about suicidal behaviour from other countries. ${ }^{8,9,12-16,18-21,23-25}$ This suggests that the use of larger samples of controls in Chinese studies would result in converting the non-significant results into statistically significant results. As mentioned above, an alternative (probably better) method for dealing with this issue would be to use dimensional assessments of personality traits rather than dichotomous measures of personality disorders.

\section{Implications}

Consistent with previous studies conducted in mainland China, ${ }^{4-6}$ the prevalence of Axis I disorders among those who died by suicide and those who attempted suicide in our study were lower than those reported in high-income countries. Using a validated measure of DSM-IV personality disorders, we also identified a much lower prevalence of personality disorders in Chinese suicide decedents, those who attempted suicide and community controls than reported elsewhere, and the prevalence remained low even after we expanded the diagnostic criteria to include both individuals with threshold and subthreshold personality disorders. The AORs (point estimates) for threshold and subthreshold personality disorders (assessed using data from proxy-informant interviews) for the suicide and suicide attempt groups (compared with the control group) were much smaller than those for Axis I mental disorders but were, nevertheless, substantial (ranging from 3.9 to 10.4), and they remained large after adjusting for comorbid Axis I mental disorders (ranging from 2.7 to 8.0). But several of these relatively large odds ratios were not statistically significant because the low prevalence of personality disorders in the control group resulted in very large confidence intervals around the estimates. We conclude that the low base rates of DSM-IV personality disorders in China seriously limits the usefulness of these dichotomous diagnostic categories in the study of the personality characteristics of individuals who take their own life and those who attempt suicide; use of continuous measures of personality traits may prove more useful for understanding the relationship between personality and suicidal behaviour. 
Yongsheng Tong, MD, PhD, Beijing Suicide Research and Prevention Center, Beijing Hui Long Guan Hospital, Beijing, WHO Collaborating Center for Research and Trainin in Suicide Prevention, Beijing, China, and Department of Psychiatry, University of Rochester Medical Center, Rochester, New York, USA; Michael R. Phillips, MD, MA $\mathrm{MPH}$, Shanghai Mental Health Center, Shanghai Jiao Tong University School of Medicine, Shanghai, China, Departments of Psychiatry and Public Health, Emory University, Atlanta Georgia, USA, and Beijing Suicide Research and Prevention Center Beijing Hui Long Guan Hospital, Beijing, China; Kenneth R. Conner, PsyD, MPH, Department of Psychiatry, University of Rochester Medical Center, Rochester, New York and US Department of Veterans Affairs VISN 2 Center of Excellence for Suicide Prevention, Canandaigua, New York, USA

Correspondence: Yongsheng Tong, Beijing Suicide Research and Prevention Center, Beijing Hui Long Guan Hospital, Beijing 100096, China. Email: timystong@pku.org.cn; Michael R. Phillips, Shanghai Mental Health Center Shanghai 201108, China. Email: mphillipschina@outlook.com

First received 19 Jul 2014, final revision 27 Jan 2015, accepted 1 Mar 2015

\section{Funding}

This study was supported by the grant from the American Foundation of Suicide Prevention (M.R.P. principal investigator (PI)). Y.T. was supported in part by PHS grants 5D43TW00910 (E.D. Caine, PI) by National Natural Science Foundation of China (NSFC, No.81371501, Beijing Municipal Science \& Technology Commission (No.Z131107002213075) and Beijing Municipal Health Bureau (QN2008-017). M.R.P. was supported in part by the National Natural Science Foundation of China (NSFC, No. 81371502). The funding institutions had no role in the design, conduct, analysis or write-up of the project.

\section{References}

1 Phillips MR, Li X, Zhang Y. Suicide rates in China, 1995-99. Lancet 2002; 359 : 835-40

2 Wang SY, Li YH, Chi GB, Xiao SY, Ozanne-Smith J, Stevenson M, et al. Injury-related fatalities in China: an under-recognized public-health problem. Lancet 2008; 372: 1765-73.

3 Tong $\mathrm{Y}$, Lan Z, XU D, Wang H, Li X, Yang C, et al. Gender differences on attempted suicide rate and fatality rate of seriously suicidal behaviors [in Chinese]. Chin J Psychiatry 2013; 46: 227-32.

4 Phillips MR, Yang G, Zhang Y, Wang L, Ji H, Zhou M. Risk factors for suicide in China: a national case-control psychological autopsy study. Lancet 2002; 360: 1728-36.

5 Zhang J, Xiao S, Zhou L. Mental disorders and suicide among young rural Chinese: a case-control psychological autopsy study. Am J Psychiatry 2010; 167: 773-81.

6 Li X, Yang R, Zhang C, Bian Q, Ji H, Wang Y, et al. A case-control study of risk factors for attempted suicide [in Chinese]. Chin J Epidemiol 2001; 22: 281-3.

7 Haw C, Hawton K, Houston K, Townsend E. Psychiatric and personality disorders in deliberate self-harm patients. Br J Psychiatry 2001; 178: 48-54.

8 Arsenault-Lapierre G, Kim C, Turecki G. Psychiatric diagnoses in 3275 suicides: a meta-analysis. BMC Psychiatry 2004; 4: 37.

9 Bertolote JM, Fleischmann A, De Leo D, Wasserman D. Psychiatric diagnoses and suicide: revisiting the evidence. Crisis 2004; 25: 147-55.

10 Nock MK, Hwang I, Sampson NA, Kessler RC. Mental disorders, comorbidity and suicidal behavior: results from the national comorbidity survey replication. Mol Psychiatry 2010; 15: 868-76.

11 Zhang J, Li N, Tu XM, Xiao S, Jia C. Risk factors for rural young suicide in China: a case-control study. J Affect Disord 2011; 129: 244-51.

12 Hawton K, Houston K, Haw C, Townsend E, Harriss L. Comorbidity of axis and axis II disorders in patients who attempted suicide. Am J Psychiatry 2003; 160: 1494-500.

13 Blasco-Fontecilla $\mathrm{H}$, Baca-Garcia E, Dervic K, Perez-Rodriguez MM Saiz-Gonzalez MD, Saiz-Ruiz J, et al. Severity of personality disorders and suicide attempt. Acta Psychiatr Scand 2009; 119: 149-55.

14 Giner L, Blasco-Fontecilla H, Perez-Rodriguez MM, Garcia-Nieto R, Giner J, Guija JA, et al. Personality disorders and health problems distinguish suicide attempters from completers in a direct comparison. J Affect Disord 2013; 151: 474-83.

15 Appleby L, Cooper J, Amos T, Faragher B. Psychological autopsy study of suicides by people aged under 35. Br J Psychiatry 1999; 175: 168-74.

16 Foster T, Gillespie K, McClelland R, Patterson C. Risk factors for suicide independent of DSM-III-R Axis I disorder. Case-control psychological autopsy study in Northern Ireland. Br J Psychiatry 1999; 175: 175-9.
17 Hawton K, Simkin S, Rue J, Haw C, Barbour F, Clements A, et al. Suicide in female nurses in England and Wales. Psychol Med 2002; 32: 239-50.

18 Portzky G, Audenaert K, van Heeringen K. Suicide among adolescents: a psychological autopsy study of psychiatric, psychosocial and personalityrelated risk factors. Soc Psychiatry Psychiatr Epidemiol 2005; 40: 922-30.

19 Schneider B, Wetterling T, Sargk D, Schneider F, Schnabel A, Maurer K, et al. Axis I disorders and personality disorders as risk factors for suicide. Eur Arch Psychiatry Clin Neurosci 2006; 256: 17-27.

20 Bolton JM, Robinson J. Population-attributable fraction of axis I and axis II mental disorders for suicide attempts: findings from a representative sample of the adult, noninstitutionalized US population. Am J Public Health 2010; 100: $2473-80$.

21 Lesage AD, Boyer R, Grunberg F, Vanier C, Morissette R, Menard-Buteau C, et al. Suicide and mental disorders: a case-control study of young men. Am J Psychiatry 1994; 151: 1063-8.

22 American Psychiatric Association. Diagnostic and Statistical Manual of Mental Disorders (4th edn) (DSM-IV). APA, 1994.

23 Vijayakumar L, Rajkumar S. Are risk factors for suicide universal? A case-control study in India. Acta Psychiatr Scand 1999; 99: 407-11.

24 Cheng AT, Mann AH, Chan KA. Personality disorder and suicide. A case-control study. Br J Psychiatry 1997; 170: 441-6.

25 Kulkarni RR, Rao KN, Bgum S. Comorbidity of psychiatric and personality disorders in first suicide attempters: a case-control study. Asian J Psychiatry 2013; 6: 410-6.

26 World Health Organization. The ICD-10 Classification of Mental and Behavioural Disorders: Clinical Descriptions and Diagnostic Guidelines. WHO, 1992.

27 Links PS, Kolla NJ, Guimond T, McMain S. Prospective risk factors for suicide attempts in a treated sample of patients with borderline personality disorder. Can J Psychiatry 2013; 58: 99-106.

28 Soloff PH, Chiappetta L. Prospective predictors of suicidal behavior in borderline personality disorders at 6-year follow-up. Am J Psychiatry 2012; 169: 484-90.

29 Yen S, Shea MT, Pagano M, Sanislow CA, Grilo CM, McGlashan TH, et al. Axis I and axis II disorders as predictors of prospective suicide attempts: findings from the collaborative longitudinal personality disorders study. J Abnorm Psychol 2003; 112: 375-81.

30 Paris J. Chronic suicidality among patients with borderline personality disorder. Psychiatry Serv 2002; 53: 738-42.

31 Beautrais AL. Suicides and serious suicide attempts: two population or one? Psychol Med 2001; 31: 837-45.

32 First MB, Spitzer RL, Gibbon M, Williams JBW. Structured Clinical Interview for DSM-IV-TR Axis I Disorders, Research Version, Patient Edition (SCID-I/P). Biometrics Research, New York State Psychiatric Institute, 2002

33 First MB, Gibbon M, Spitzer RL, Williams JBW, Benjamin LS. Structured Clinical Interview for DSM-IV Axis II Personality Disorders (SCID-II). American Psychiatric Press, 1997.

34 Dai Y, Xiao Z, Wang Z. The reliability and validity of a Chinese version of the Structured Clinical Interview for DSM-IV personality disorders [in Chinese]. Shanghai Arch Psychiatry 2006; 18: 1-4.

35 Tyrer $P$, Johnson $T$. Establishing the severity of personality disorder. Am J Psychiatry 1996; 153: 1593-7.

36 Yang G, Phillips MR, Zhou M, Wang L, Zhang Y, Xu D. Understanding the unique characteristics of suicide in China: national psychological autopsy study. Biomed Environ Sci 2005; 18: 379-89.

37 Conner KR, Phillips MR, Meldrum SC. Predictors of low-intent and high-intent suicide attempts in rural China. Am J Public Health 2007; 97: 1842-6.

38 Conner KR, Phillips MR, Meldrum S, Knox KL, Zhang Y, Yang G. Low-planned suicides in China. Psychol Med 2005; 35: 1197-204.

39 Trull TJ. Dimensional models of personality disorder. Curr Opin Psychology 2000; 13: 179-84.

40 Frances AJ, Nardo JM. ICD-11 should not repeat the mistakes made by DSM-5. Br J Psychiatry 2013; 203: 1-2.

41 American Psychiatric Association. Diagnostic and Statistical Manual of Mental Disorders (5th edn) (DSM-5). APA, 2013.

42 Phillips MR, Zhang J, Shi Q, Song Z, Ding Z, Pang S, et al. Prevalence, associated disability and treatment of mental disorders in four provinces in China, 2001-2005: an epidemiological survey. Lancet 2009; 373: 2041-53.

43 Crump C, Sundquist K, Winkleby MA, Sundquist J. Mental disorders and risk of accidental death. Br J Psychiatry 2013; 203: 297-302. 